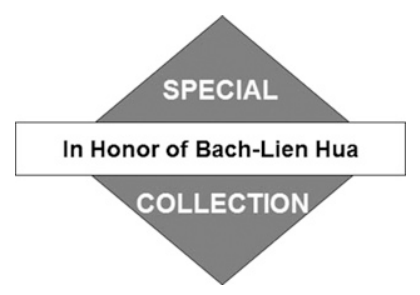

\title{
Cyclones and Anticyclones in Seismic Imaging
}

\author{
A. C. Barbosa Aguiar,* C. Ménesguen, S. Le Gentil, and R. Schopp \\ Laboratoire de Physique des Océans, IFREMER, Brest, France \\ X. CARTON \\ Laboratoire de Physique des Océans, UBO/UEB, Brest, France
}

(Manuscript received 2 April 2015, in final form 24 June 2015)

\begin{abstract}
Nearly all the subsurface eddies detected in seismic imaging of sections in the northeast Atlantic have been assumed to be anticyclones containing Mediterranean Water (MW). Fewer MW cyclones have been observed and studied. In this study, the work of previous numerical studies is extended to investigate some characteristics of layering surrounding MW cyclones, using a primitive equation model with equal diffusivities for salinity and temperature to suppress the effects of double diffusion. It is shown that, after a stable state is reached, both anticyclones and cyclones display similar patterns of layering: stacked thin layers of high acoustic reflectivity located above and below the core of each vortex, which do not match isopycnals. The authors conclude that it should not be possible to distinguish between MW cyclones and anticyclones based on their signature in seismic imaging alone. Complementary information is needed to determine the sense of rotation.
\end{abstract}

\section{Introduction}

Subsurface vortices containing Mediterranean Water (MW; warm and salty) can easily be identified in seismic imaging because of the strong small-scale temperature gradient observed on their periphery (Gonella and Michon 1988; Holbrook et al. 2003; Nandi et al. 2004; Biescas et al. 2008).

The vast majority of these structures have clockwise rotation, and the term meddy is widely used to refer to an anticyclonic eddy of MW. Nonetheless, several observations have put in evidence the ubiquity of cyclonic eddies often coupled with meddies (Richardson et al. 2000; Carton et al. 2002; L'Hégaret et al. 2014). These cyclones are regularly formed off the southern coast of Portugal in the MW layers (Serra et al. 2005; Barbosa Aguiar et al. 2013).

\footnotetext{
* Current affiliation: Instituto Dom Luiz, Faculdade de Ciências, Universidade de Lisboa, Lisbon, Portugal.

Corresponding author address: A. C. Barbosa Aguiar, Instituto Dom Luiz, Faculdade de Ciências, Universidade de Lisboa, Campo Grande, 1749-016 Lisbon, Portugal.

E-mail: aaaguiar@fc.ul.pt
}

In situ studies of cyclone-meddy dipoles near the Portimão Canyon revealed that the cyclonic part also bears anomalies in temperature $T$ and salinity $S$, albeit weaker than its companion meddy. In the example surveyed by Carton et al. (2002), maximal $T$ and $S$ within the cyclone were of $12^{\circ} \mathrm{C}$ and $36.2 \mathrm{psu}$, whereas in the meddy maxima values were of $12.5^{\circ} \mathrm{C}$ and $36.5 \mathrm{psu}$. In the same study, the thermohaline and velocity cores of the cyclone were located between 600 and $1300 \mathrm{~m}$, at shallower depths than those of the meddies $(750-1500 \mathrm{~m})$, while the radii of the structures were comparable and about $30-35 \mathrm{~km}$.

In images of acoustic reflectivity of the water column, meddies appear imprinted as distinct elliptical contours because of the existence of thin, stacked (pancake-like), highly reflective layers surrounding their cores (layering). So far, most of subsurface eddies identified in seismic sections in the northeast Atlantic have been interpreted as anticyclones. However, in the western end of the Iberian-Atlantic Margin (IAM) seismic acquisition survey section IAM-5, Pinheiro et al. (2010) identified an MW cyclonic vortex (see Fig. 1) based on simultaneous drifter's data that show a counterclockwise looping trajectory partly coincident with IAM-5. This MW cyclone had already been identified by Richardson et al. (2000), who 


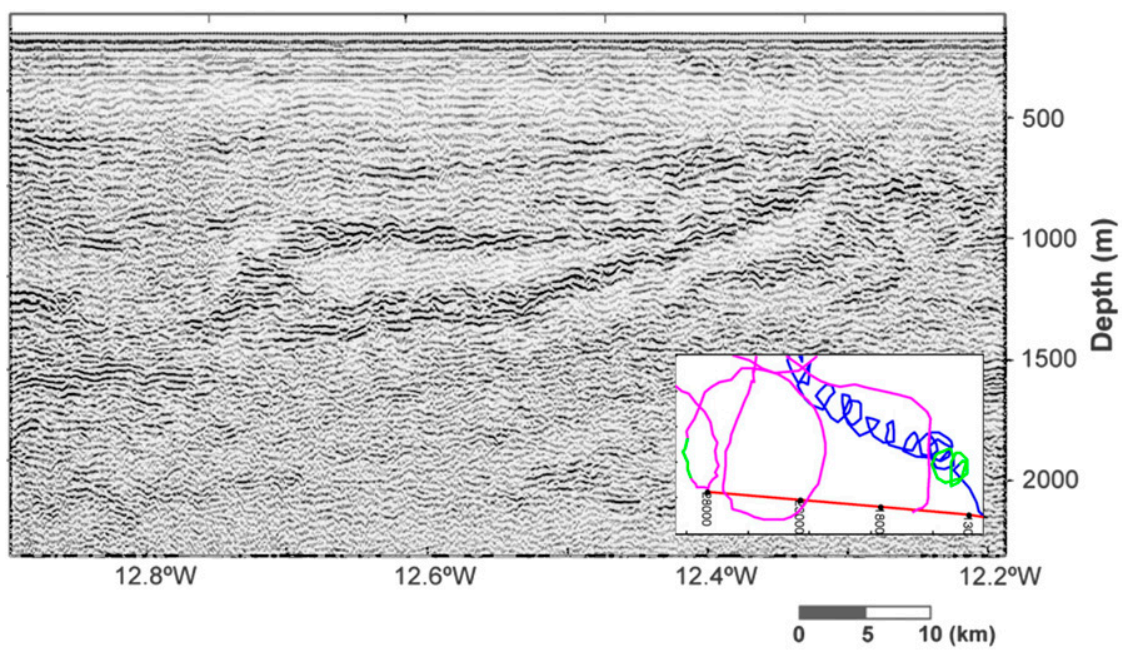

FIG. 1. MW cyclone in seismic section IAM-5, identified with the help of overlapping drifter data previously reported by Richardson et al. (2000) as corresponding to an MW cyclone. The inset shows the seismic section in red and the drifter's trajectory in magenta, switching to green over the time period coinciding with the seismic survey. Adapted with written permission from Pinheiro et al. (2010).

analyzed a large dataset of drifter records in the Iberian and Canarian basins to compile an observational meddy census. Can one recognize an MW cyclone lens as such just from its signature in seismic imaging?

On the one hand, geostrophic and hydrostatic balances dictate a different mean density structure for anticyclones and cyclones: the strongest density gradient is located at middepth in a cyclone whereas it is confined to the core's periphery in a meddy. This means that the isopycnals of anticyclones are shaped as a convex lens, whereas those of cyclones are instead as a concave lens. On the other hand, streamfunctions of either vortex are similar aside from being of opposite signs.

Depending on the dominant dynamical processes responsible for the observed layering, the inherent differences between cyclones and anticyclones may or not translate into a unique signature of each vortex type in seismic imaging.

Several authors have proposed different mechanisms to explain the formation of layering. Meunier et al. (2015) showed that the thin layers seen in seismic imaging can arise because of isopycnal stirring by the vortex velocity field. Layering can also be created by the baroclinic instability of meddy-type vortices as shown by different studies in a quasigeostrophic (QG) framework (Nguyen et al. 2012; Ménesguen et al. 2012; Hua et al. 2013). To date, the formation of layering has always been studied considering an intrinsically symmetric framework with respect to the sense of rotation of the vortex. In other words, in the previous studies, a cyclone would stir tracers and/or develop the same QG instabilities as an anticyclone, always evolving toward a convex seismic lens shape. To investigate if a cyclone can develop a distinct signature in acoustic reflectivity, we must use a model that allows vortices of opposite sign to evolve differently.

In this study we use a primitive equation (PE) model to investigate two isolated and idealized weakly perturbed vortices: an anticyclone and a cyclone. In the model, the initial velocity profiles are identical (apart from the sense of rotation) and in thermal wind balance. The vortex evolution is time-integrated as a freely evolving system until reaching a state when layering is developed. We present a comparison of the layering pattern observed in each case.

It should be noted that not all mechanisms that may explain the formation of layering are included in our idealized simulations. For example, double diffusion is often cited to explain the thermohaline staircases found above and below meddies (e.g., Ruddick and Hebert 1988; Ruddick 1992). In this paper, we have decided to address only the dynamics of stirring and baroclinic instability and their possible impact on the layering patterns observed in seismic imaging. The role of double diffusion is beyond the scope of our present numerical study where the diffusivities for salinity and temperature are taken as equal.

\section{Model}

The model is a PE model, with constant background stratification, that uses the finite difference method in all spatial directions. It was developed by H. Aiki (JAMSTEC) and is fully described in Aiki and Yamagata (2006) and Aiki et al. (2011). The results presented here correspond to 
simulations in a grid of $512 \times 512 \times 480$ points with a resolution of $400 \mathrm{~m}$ in the horizontal plane.

The hydrostatic and incompressible Boussinesq equations are implemented on the $f$ plane using a flat-bottom, free-slip configuration; a free surface with implicit scheme (Dukowicz and Smith 1994); and open radiative lateral boundary conditions. The advection scheme is a third-order upwind scheme for momentum and tracers. In the horizontal, dissipation, and diffusion are implicit because of the advection scheme. Vertical momentum dissipation and tracer diffusivity are both modeled by a biharmonic operator with a coefficient of $3.05 \times$ $10^{-5} \mathrm{~m}^{4} \mathrm{~s}^{-1}$, for a vertical grid spacing of $6.25 \mathrm{~m}$. Therefore, diffusivities for momentum and density are equal (Prandtl number is 1), suppressing McIntyre's instability (McIntyre 1970). The time integration follows a leapfrog scheme with the Matsuno scheme blended every five time steps. The baroclinic time step is $20 \mathrm{~s}$. No explicit forcing is applied.

The initial vortex profile is a solution of the QG dynamics and therefore will be weakly unstable in the PE framework. Small random perturbations are initially added to the energy of the system in order to accelerate the slow process of baroclinic instability.

\section{Initial vortex profiles}

Previous work concerning the anticyclonic case (Hua et al. 2013) showed that the pattern of layering that evolves is robust and does not depend on the shape chosen for the initial vortex profile. For the sake of simplicity, we chose a Gaussian shape (as in Nguyen et al. 2012) for the streamfunction of the initial vortex:

$$
\psi(x, y, z)=A \exp \left[-\frac{x^{2}}{L^{2}}-\frac{y^{2}}{L^{2}}-\frac{\left(z-z_{0}\right)^{2}}{H^{2}}\right] .
$$

The vortex is centered at $\left(0,0, z_{0}\right)$, having horizontal and vertical length scales of $L$ and $H$, respectively. Initial velocities are purely horizontal, $(u, v)=(-\partial \psi / \partial y, \partial \psi / \partial x)$, with amplitudes proportional to $A$ ( $A$ is a constant; $A=$ $4500 \mathrm{~m}^{2} \mathrm{~s}^{-1}$ ).

To ensure a negligible influence of the top and bottom boundaries, the vortex center is positioned at $1500 \mathrm{~m}$ $\left(z_{0}\right)$, deeper than observed in the ocean, and the domain is $3000 \mathrm{~m}$ deep.

Dynamically, a vortex is characterized by two nondimensional parameters: the Burger number $\mathrm{Bu}=\left(N H / f_{0} L\right)^{2}$ and the Rossby number Ro $=\nabla_{h}^{2} \psi(0,0) / f_{0}=-4 A /\left(f_{0} L^{2}\right)$. The Coriolis parameter and the Brunt-Väisälä frequency are chosen to be $f_{0}=8 \times 10^{-5} \mathrm{~s}^{-1}$ and $N=2.24 \times 10^{-3} \mathrm{~s}^{-1}$, respectively, typical values for the area off southwest
Iberia (Hua et al. 2013). Here, $\mathrm{Bu}=0.12$ and $|\mathrm{Ro}|=0.22$ (Ro is negative in the anticyclone), for $H=400 \mathrm{~m}$ and $L=$ $32 \mathrm{~km}$, characteristic length scales for meddies.

The density is defined as a linear function of $S$ and $T$,

$$
\rho=\rho_{0}\left[1+\beta\left(S-S_{0}\right)-\alpha\left(T-T_{0}\right)\right],
$$

where $\rho_{0}=1027 \mathrm{~kg} \mathrm{~m}^{-3}, S_{0}=36.6 \mathrm{psu}$, and $T_{0}=13^{\circ} \mathrm{C}$ are the density, salinity, and temperature values, respectively, corresponding to the saltier and warmer water trapped within the vortex. The values of the coefficients of thermal expansion and saline contraction are $\alpha=1.6 \times 10^{-4}{ }^{\circ} \mathrm{C}^{-1}$ and $\beta=7.8 \times 10^{-4} \mathrm{psu}^{-1}$.

The choice of parameter values is based on a comparison of the analytically derived velocity profiles with those of meddies reproduced by a realistic long-term numerical simulation (Barbosa Aguiar et al. 2013; see Fig. 2). Note that the anomalies of the chosen temperature and salinity profiles largely compensate in the final density profile (see Fig. 2e).

The discrepancy between the analytical profiles and those of a realistic simulation can be partly explained by the approximations chosen to simplify our problem. The vortex initialization assumes hydrostatic and geostrophic balance, neglecting the cyclostrophic term. Moreover, the velocity amplitude had to be kept small because a Gaussian vortex with the chosen vertical scale but larger velocities exhibits a statically unstable core.

\section{Results: Anticyclone versus cyclone}

After 100 days of simulation, the idealized vortices become unstable and develop filaments that spiral outwards from the core. In the cyclone's case, the spiral arms appear 10 days earlier and quickly become deformed and asymmetric, detaching from the main core around day 150. In the anticyclone, the spiral arms remain axisymmetric until day 180 and detach shortly after 200 days. Similar numerical studies such as those reported by Hua et al. (2013) and Meunier et al. (2015) provide a detailed analysis of the time evolution of a weakly perturbed baroclinic (anticyclonic) vortex.

Figures $3 \mathrm{a}$ and $3 \mathrm{~b}$ show a horizontal section of the vorticity of each vortex at day 270 . In both cases, the main vortex has an axisymmetric shape and is surrounded by satellite vortices and filaments.

After the first stage of evolution, the main vortex exhibits layering that is intensified in fields of the acoustic reflectivity as illustrated in Figs. 3c and 3d. Here we show the signal corresponding to vertical scales smaller than $100 \mathrm{~m}$. This is obtained by subtracting from the original acoustic impedance field a moving average over $100 \mathrm{~m}$ in the vertical direction. It corresponds to what is observed 


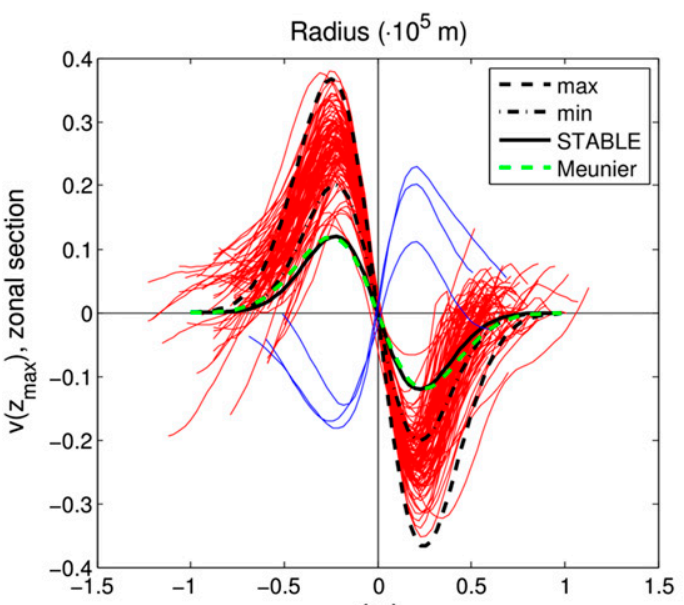

(a)

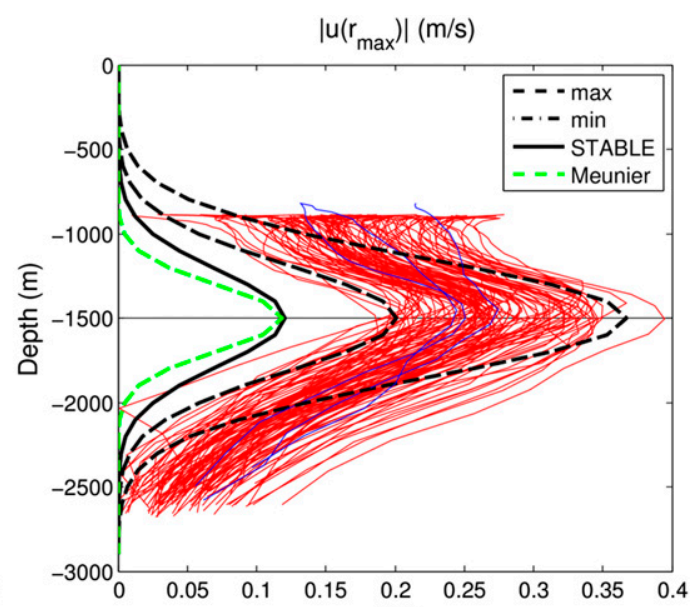

(b)

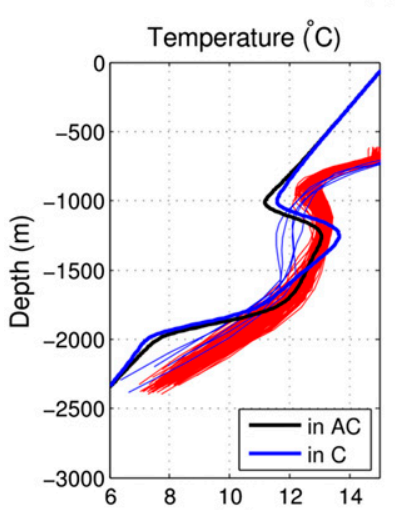

(c)

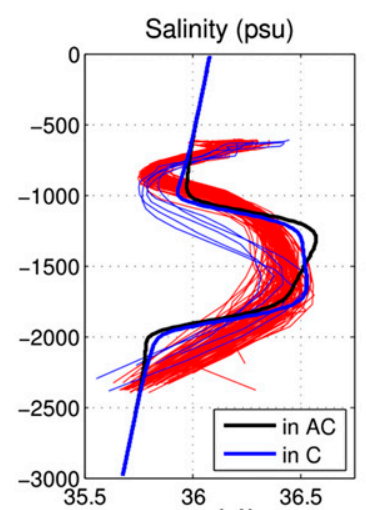

(d)

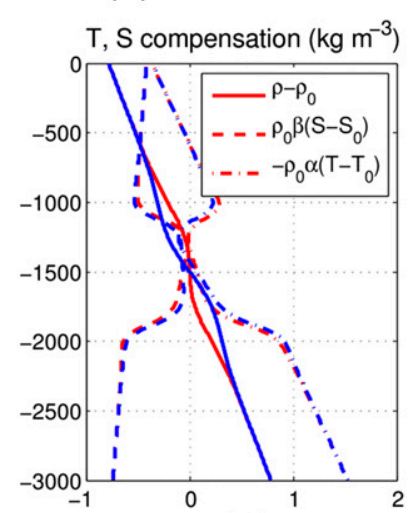

(e)

FIG. 2. Initial profiles of (a),(b) velocity, (c) temperature, (d) salinity, and (e) density. Thin lines are "realistic" profiles obtained from the simulation in Barbosa Aguiar et al. (2013). Anticyclones are in red, cyclones are in blue. These correspond to time averages between days 30 and 60 of the meddy's lifetime. The azimuthal velocity profiles $v\left(r, z_{\max }\right)$ and $u\left(r_{\max }, z\right)$ correspond to the maximum swirl speed. The temperature and salinity vertical profiles were taken at zero radius. The black solid line represents the statically stable Gaussian profile used in the present study. The green dashed line is the Gaussian profile corresponding to the parameter values used by Meunier et al. (2015). The black dashed and dash-dotted lines are Gaussian profiles that are both statically unstable.

in seismic surveys using an acoustic source with a lowerbound frequency of $\sim 15 \mathrm{~Hz}$. For acoustic media like water, the impedance is defined as the product of compressional sound speed and density (Papenberg et al. 2010). The acoustic reflectivity is approximately the vertical derivative of impedance and is mostly proportional to the gradient of temperature convoluted with the source signal.

The synthesized seismic signal in Figs. $3 \mathrm{c}$ and $3 \mathrm{~d}$ presents layering features that are similar to what is observed in seismic imaging: regions of contrasting bright and dark shades correspond to the characteristic stacks of reflectors visible in seismic imaging (e.g., Biescas et al. 2008).

Since the acoustic reflectivity is primarily due to vertical gradients of thermal perturbations (e.g., Nandi et al. 2004; Biescas et al. 2014), the signature of layering in $d T^{\prime} / d z$ looks very similar to that of acoustic impedance as seen in Fig. 4.
It is worth noticing that the bright/dark layers do not follow the shape of the isopycnals (cyan lines in Figs. 3c,d) but lie closer to the streamline contours (yellow lines in Fig. 4). This is even more flagrant for a cyclonic vortex where the layering pattern is convex but the isopycnals are concave.

The most striking result is the absence of any significant differences between the fine structures observed in each case. In the cyclone, the layering is concentrated above and below the core at the same location as already demonstrated for anticyclones (Ménesguen et al. 2009; Hua et al. 2013; Meunier et al. 2015). Along the edges of secondary vortices and filaments there is a strong signature of layering as well.

The work of Meunier et al. (2015) strongly suggests that the stacked reflectors are primarily due to tracer stirring along the streamlines of a vortex field, not excluding the 


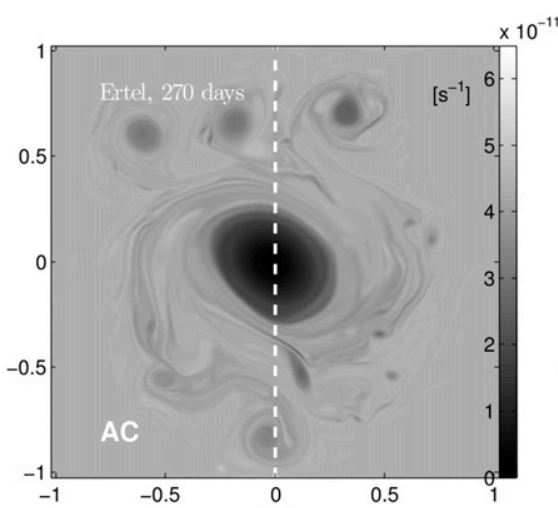

(a)

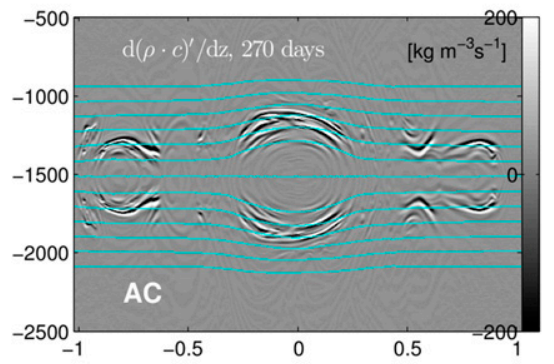

(c)

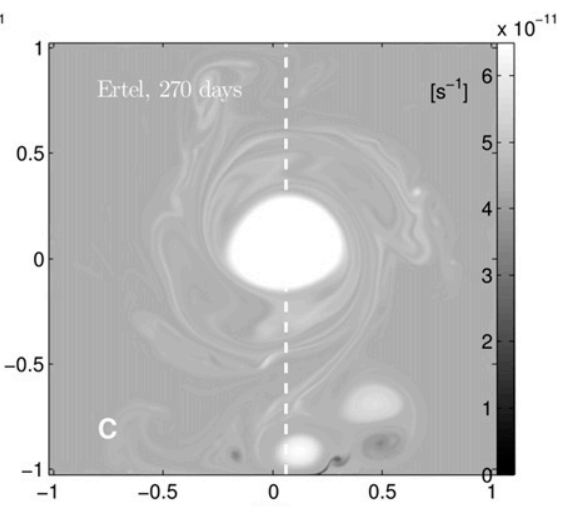

(b)

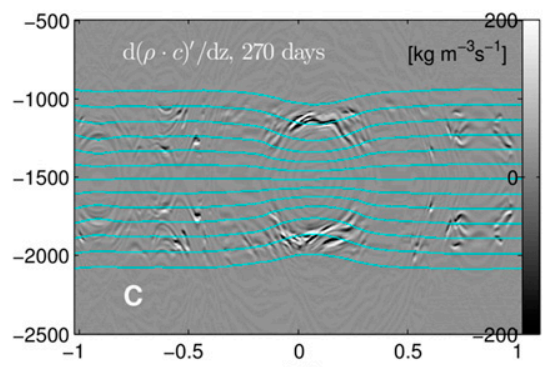

(d)

FIG. 3. Model results for the (a),(c) anticyclone and (b),(d) cyclone. Horizontal section of Ertel's potential vorticity at (top) middepth and (bottom) layering signal in acoustic reflectivity with overlaid isopycnal contours across the vertical section indicated by the dashed line in the corresponding Ertel's field. Isopycnals are depicted for values between 1026.7 and 1027.3 with a contour interval of 0.05 . The value of $d(\rho c)^{\prime} / d z$ was computed after subtracting a moving average (over $100 \mathrm{~m}$ in the vertical) from the values of $\rho c$, where $c=c(z, T, S)$ is the speed of sound.

possibility of other concomitant processes, such as baroclinic instability (Nguyen et al. 2012). The results presented here support and highlight these hypotheses.

\section{Dynamical layering processes}

In Fig. 5 the vertical profiles of perturbations in spice and velocity $\left(u^{\prime}, v^{\prime}\right)$ are given along with vertical mean shear $\left(\partial_{z} \bar{u}, \partial_{z} \bar{v}\right)$ and radial gradient of mean Ertel potential vorticity $\left(\bar{q}_{E}\right)$. Spice is defined as sp $=\rho_{0}(\alpha T+\beta S)$ and acts as a passive tracer, being commonly used to study water masses where temperature and salinity have a compensating effect. These profiles correspond to a location at about $12 \mathrm{~km}$ away from the center of the vortex in the cross section represented by the dashed line in Figs. 3a and $3 \mathrm{~b}$. Away from the center the velocities are significant.

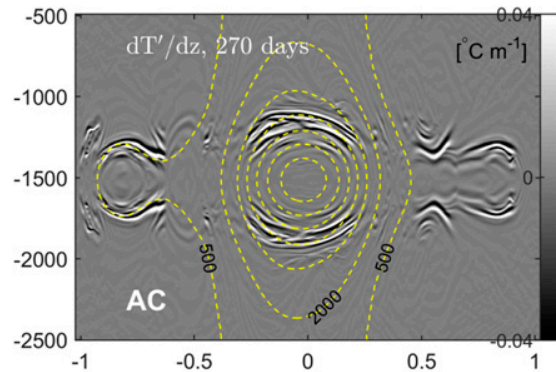

(a)

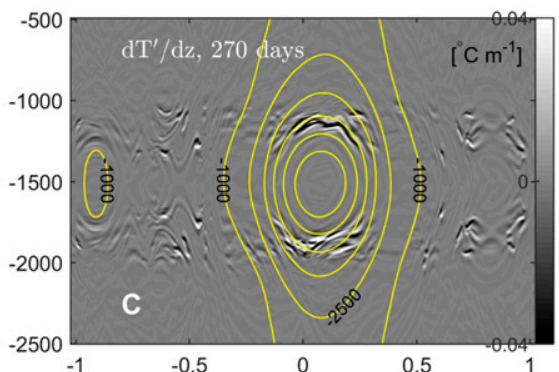

(b)

FIG. 4. Model results for the (a) anticyclone and (b) cyclone: layering signal in $d T^{\prime} / d z$ across the vertical section indicated by the dashed lines in the corresponding Ertel's field in Figs. 3a and $3 \mathrm{~b}$. Streamlines are depicted with a contour interval of $1500 \mathrm{~m}^{2} \mathrm{~s}^{-1}$, dashed/solid lines correspond to positive/negative values. The value of $d T^{\prime} / d z$ was computed after subtracting a moving average (over $100 \mathrm{~m}$ in the vertical) from the temperature values. 

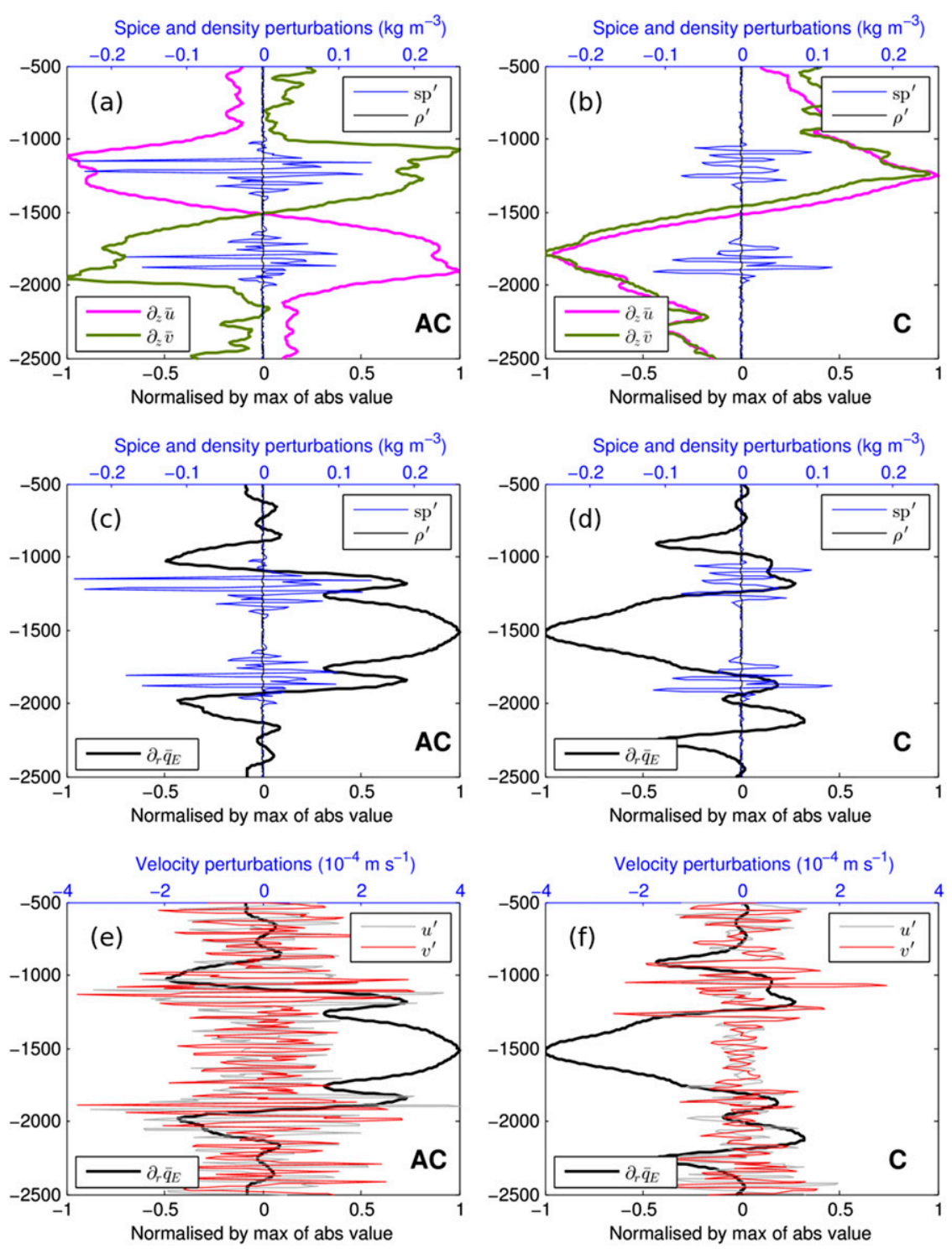

FIG. 5. Profiles in the (a),(c),(e) anticyclone and (b),(d),(f) cyclone at $12 \mathrm{~km}$ away from the center of the vortex. A prime is used to denote a perturbation whenever a profile is filtered, retaining only scales smaller than $100 \mathrm{~m}$, using a high-pass Butterworth filter of order 2 for velocity $(u, v)$, spice $(\mathrm{sp})$, and density $(\rho)$. A bar is used to denote a mean profile, that is, the total subtracted of the respective perturbation. The thick lines correspond to $\partial_{z} \bar{u}, \partial_{z} \bar{v}$, and $\partial_{r} \bar{q}_{E}$, normalized by the respective maximum of its absolute value.

Primed quantities refer to small-scale perturbations obtained after using a high-pass Butterworth filter of order 2 with a cutoff length scale of $100 \mathrm{~m}$. A bar denotes a mean profile, that is, the original profile subtracted of small-scale perturbations, for example, $\bar{u}(z)=u(z)-u^{\prime}(z)$.

Figures $5 \mathrm{a}$ and $5 \mathrm{~b}$ show that layering is present in spice anomalies (small-scale variations) above and below the vortex where the vertical mean shear is greatest. This result is consistent with the tracer stirring hypothesis suggested by Meunier et al. (2015).
On the other hand, Nguyen et al. (2012) and Hua et al. (2013) concluded that growing perturbations at critical layers around the vortices can also produce layering. A necessary condition for the development of baroclinic instability is that the gradient of $\bar{q}_{E}$ must change sign across the domain (Rayleigh-Kuo-Fjørtoft criterion; Eliassen 1983). In Figs. 5c and 5d we plot the radial gradient of $\bar{q}_{E}$ verifying that, to a first approximation, the aforementioned condition prevails at the location where layering occurs. The criterion is satisfied in both 
case studies, suggesting that the layering exhibits a signature of baroclinic instability processes.

In addition, in Figs. 5e and $5 \mathrm{f}$ we observe that the velocity profiles also exhibit a layering structure. The amplitude of this layering is greater where $\bar{q}_{E}$ gradient changes sign. Such a feature cannot be due to pure tracer stirring but can instead be an attribute of ongoing baroclinic instability, known to be intense in the critical levels (Nguyen et al. 2012; Meunier et al. 2015).

The small differences between the profiles computed for anticyclone and cyclone are due to small-scale noise and cannot be used to distinguish between the two.

To summarize, Fig. 5 indicates that while stirring may play a leading role toward layering, baroclinic instability can also be important.

\section{Discussion and conclusions}

In this study, we have weakly perturbed two vortices (a cyclone and an anticyclone) containing warm and salty water. The system of equations describing their evolution was time integrated until layering could be detected in the acoustic impedance signal of the vortices.

Initially, the difference between the two opposite rotation cases resides in the inherent density structure. The cyclonic lens becomes unstable about 10 days earlier than the anticyclonic lens, in our primitive equations' simulations. After 200 days, the layering pattern displayed by each vortex is similar except for minor details. In the anticyclone, the thin layers tend to be smooth and steady whereas in the cyclone, the layers are more undulating and irregular (time evolution not shown).

We conclude that the pattern of fine-scale structures surrounding the core of a cyclone resembles that of anticyclones. There are no significant differences in position, shape, or size of the thin layers. Therefore, it does not seem possible to distinguish anticyclones from cyclones based on seismic imaging alone.

This work also shows that similar perturbations occur in the velocity profiles, despite the fact that only tracer layering can be detected in seismic images.

Furthermore, the highly reflective layers are not collocated with density interfaces and the discrepancy between isopycnals and seismic reflection isolines is very large in the case of a cyclonic vortex. We show that there is no direct relationship between highly reflective layers and isopycnals.

In the case of MW eddies, the physical characteristics such as preferred depth and length scales of cyclones and anticyclones are not distinct enough to be reliable indicators of the sense of rotation in a seismic image. However, information on the dynamics of the structures observed can be derived from a thorough analysis of seismic data. The velocity of the vortex (along the seismic section, within the limits of geostrophy) can be determined after a reconstruction of the density field. To retrieve the density field from seismic data, Papenberg et al. (2010) and Biescas et al. (2014) have successfully applied the full waveform inversion method to seismic data combined with in situ profiles of temperature and salinity. Bornstein et al. (2013) explored inversion methods that should not need simultaneous oceanographic data.

Seismic section IAM-5 appears to have crossed the southern edge of an MW cyclone (Richardson et al. 2000; Pinheiro et al. 2010). This feature went unnoticed in an earlier study of that very same seismic section (Buffett et al. 2009). To date, this is the only report of an MW cyclone observation in seismic data, and its identification was only possible thanks to simultaneous drifter records within the same structure.

We conclude that a competition between stirring and baroclinic instability may exist in layering formation. Although double diffusion was not taken into account in our study, it is another mechanism likely to be relevant in the layering process and may compete with the former two. Further studies are required to investigate this.

Acknowledgments. This study was made possible through the support of Grant "Ocean Layering" (PDOC 2011) of Agence Nationale de la Recherche and project 96130 of Institut du Developpement et des Ressources en Informatique Scientifique. The authors are grateful to $\mathrm{H}$. Aiki for providing the source code of his numerical model.

\section{REFERENCES}

Aiki, H., and T. Yamagata, 2006: Energetics of the layer-thickness form drag based on an integral identity. Ocean Sci., 2, 161-171, doi:10.5194/os-2-161-2006.

_ J. P. Matthews, and K. G. Lamb, 2011: Modeling and energetics of tidally generated wave trains in the Lombok Strait: Impact of the Indonesian Throughflow. J. Geophys. Res., 116, C03023, doi:10.1029/2010JC006589.

Barbosa Aguiar, A., A. Peliz, and X. Carton, 2013: A census of Meddies in a long-term high-resolution simulation. Prog. Oceanogr., 116, 80-94, doi:10.1016/j.pocean.2013.06.016.

Biescas, B., V. Sallarès, J. L. Pelegrí, F. Machín, R. Carbonell, G. Buffett, J. J. D. Nobeitia, and A. Calahorrano, 2008: Imaging meddy finestructure using multichannel seismic reflection data. Geophys. Res. Lett., 35, L11609, doi:10.1029/ 2008GL033971.

— B. R. Ruddick, M. R. Nedimovic, V. Sallarès, G. Bornstein, and J. F. Mojica, 2014: Recovery of temperature, salinity, and potential density from ocean reflectivity. J. Geophys. Res. Oceans, 119, 3171-3184, doi:10.1002/2013JC009662.

Bornstein, G., B. Biescas, V. Sallarès, and J. F. Mojica, 2013: Direct temperature and salinity acoustic full waveform inversion. Geophys. Res. Lett., 40, 4344-4348, doi:10.1002/grl.50844. 
Buffett, G. G., B. Biescas, J. L. Pelegrí, F. Machín, V. Sallarès, R. Carbonell, D. Klaeschen, and R. Hobbs, 2009: Seismic reflection along the path of the Mediterranean undercurrent. Cont. Shelf Res., 29, 1848-1860, doi:10.1016/j.csr.2009.05.017.

Carton, X., L. Chérubin, J. Paillet, Y. Morel, A. Serpette, and B. L. Cann, 2002: Meddy coupling with a deep cyclone in the Gulf of Cadiz. J. Mar. Syst., 32, 13-42, doi:10.1016/ S0924-7963(02)00028-3.

Dukowicz, J. K., and R. D. Smith, 1994: Implicit free-surface method for the Bryan-Cox-Semtner ocean model. J. Geophys. Res., 99, 7991-8014, doi:10.1029/93JC03455.

Eliassen, A., 1983: The Charney-Stern theorem on barotropicbaroclinic instability. Pure Appl. Geophys., 121, 563-572, doi:10.1007/BF02590155.

Gonella, J., and D. Michon, 1988: Ondes internes profondes révélées par sismique réflexion au sein des masses d'eau en Atlantique-Est. C. R. Acad. Sci. Paris Ser. II, 306, 781-787.

Holbrook, W., P. Páramo, S. Pearse, and W. Schmitt, 2003: Thermohaline fine structure in an oceanographic front from seismic reflection profiling. Science, 301, 821-824, doi:10.1126/science.1085116.

Hua, L. B., C. Ménesguen, S. L. Gentil, R. Schopp, B. Marsset, and H. Aiki, 2013: Layering and turbulence surrounding an anticyclonic oceanic vortex: In situ observations and quasigeostrophic numerical simulations. J. Fluid Mech., 731, 418442, doi:10.1017/jfm.2013.369.

L'Hégaret, P., and Coauthors, 2014: Evidence of Mediterranean water dipole collision in the Gulf of Cadiz. J. Geophys. Res Oceans, 119, 5337-5359, doi:10.1002/2014JC009972.

McIntyre, M. E., 1970: Diffusive destabilization of the baroclinic circular vortex. Geophys. Fluid Dyn., 1, 19-57, doi:10.1080/ 03091927009365767.

Ménesguen, C., B. L. Hua, C. Papenberg, D. Klaeschen, L. Géli, and R. Hobbs, 2009: Effect of bandwidth on seismic imaging of rotating stratified turbulence surrounding an anticyclonic eddy from field data and numerical simulations. Geophys. Res. Lett., 36, L00D05, doi:10.1029/2009GL039951.

_, —, X. Carton, F. Klingelhoefer, P. Schnurle, and C. Reichert, 2012: Arms winding around a meddy seen in seismic reflection data close to the Morocco coastline. Geophys. Res. Lett., 39, L05604, doi:10.1029/2011GL050798.

Meunier, T., C. Ménesguen, R. Schopp, and S. L. Gentil, 2015: Tracer stirring around a meddy: The formation of layering. J. Phys. Oceanogr., 45, 407-423, doi:10.1175/ JPO-D-14-0061.1.

Nandi, P., W. S. Holbrook, S. Pearse, P. Páramo, and R. W. Schmitt, 2004: Seismic reflection imaging of water mass boundaries in the Norwegian Sea. Geophys. Res. Lett., 31, L23311, doi:10.1029/2004GL021325.

Nguyen, H. Y., B. L. Hua, R. Schopp, and X. Carton, 2012: Slow quasigeostrophic unstable modes of a lens vortex in a continuously stratified flow. Geophys. Astrophys. Fluid Dyn., 106, 305-319, doi:10.1080/03091929.2011.620568.

Papenberg, C., D. Klaeschen, G. Krahmann, and R. W. Hobbs, 2010: Ocean temperature and salinity inverted from combined hydrographic and seismic data. Geophys. Res. Lett., 37, L04601, doi:10.1029/2009GL042115.

Pinheiro, L. M., H. Song, B. Ruddick, J. Dubert, I. Ambar, K. Mustafa, and R. Bezerra, 2010: Detailed 2-D imaging of the Mediterranean outflow and meddies off W Iberia from multichannel seismic data. J. Mar. Syst., 79, 89-100, doi:10.1016/ j.jmarsys.2009.07.004.

Richardson, P. L., A. Bower, and W. Zenk, 2000: A census of meddies tracked by floats. Prog. Oceanogr., 45, 209-250, doi:10.1016/S0079-6611(99)00053-1.

Ruddick, B., 1992: Intrusive mixing in a Mediterranean salt lensIntrusion slopes and dynamical mechanisms. J. Phys. Oceanogr., 22, 1274-1285, doi:10.1175/1520-0485(1992)022<1274: IMIAMS $>2.0 . \mathrm{CO} ; 2$. , and D. Hebert, 1988: The mixing of Meddy "Sharon." SmallScale Turbulence and Mixing in the Ocean, J. C. J. Nihoul, and B. M. Jamart, Eds., Elsevier Oceanography Series, Vol. 46, Elsevier, 249-261, doi:10.1016/S0422-9894(08)70551-8.

Serra, N., I. Ambar, and R. Käse, 2005: Observations and numerical modelling of the Mediterranean outflow splitting and eddy generation. Deep-Sea Res. II, 52, 383-408, doi:10.1016/ j.dsr2.2004.05.025. 